\title{
THE CONSEQUENCES OF MUTATION IN MULTI-CLONAL ASEXUAL SPECIES
}

\author{
J. T. MANNING \\ Institute of Extension Studies, University of Liverpool, Liverpool, U.K.
}

Received 19.vii.82

\section{SUMMARY}

\begin{abstract}
The consequences of favourable and deleterious mutations in an asexual population with two alleles $A$ and $a$ are considered. The frequency of $A=p$ and that of $a=q, p \neq q$ is likely so that $p>q$ is assumed. The polymorphism is maintained by frequency dependent selection. In such a species there will be a tendency for advantageous mutations to accumulate most rapidly in clone $A$ and deleterious mutations to accumulate most rapidly in clone $a$. Eventually the species will become monomorphic for $A$. During the process clone $A$ will become larger but will lose fitness. Sex prevents clonal loss and permits the existence of intense variation.
\end{abstract}

\section{INTRODUCTION}

Most species reproduce sexually. Despite this there appear to be a number of substantial disadvantages associated with sex and it is poorly understood why parthenogenesis is not more common (Maynard Smith, 1978). Most models constructed by population geneticists address themselves to sexual species. The consequences of mutation in sexual populations are therefore well understood. In order to understand the advantages of sex we must concentrate our thoughts on the consequences of asexual reproduction. The existence of some parthenogenetic species indicates that asexual reproduction does have some advantages over sex in certain conditions. For example in colonising species or those that experience frequent drastic reductions in numbers, parthenogenesis may provide an escape from the deleterious effects of inbreeding (Manning, 1981). We are concerned here, however, with relatively stable populations which are initially assumed to be polymorphic. For simplicity we assume an asexual haploid population of $N$ individuals which is polymorphic for two alleles $A$ and $a$, with equilibrium frequencies $p$ and $q$. The polymorphism is maintained by frequency dependent selection. It is likely that $p \neq q$ so that $p>q$ is assumed. What are the consequences of favourable and deleterious mutations in such a species?

\section{FAVOURABLE MUTATIONS}

Suppose the species is monomorphic for gene $b$. A change in the environment occurs so that the mutation $b \rightarrow B$ is favoured in both clones. The species now experiences a genetic load which has been called a lag load by Maynard Smith (1976). Genetic load is conventionally designated as

$$
\frac{\left(W_{\max }-\bar{W}\right)}{W_{\max }}
$$


with $W_{\max }=$ the maximum fitness the population can achieve and $\bar{W}=$ the actual mean fitness of the population. If $B$ is fixed $\bar{W}=1$, but as $b$ is at present at fixation $\bar{W}=1-s$. The lag load arises because the population is not optimally adapted to the environment and is

$$
\frac{(1-(1-s))}{1} \text {. }
$$

Now suppose $b \rightarrow B$ occurs. Manning (1982a) has pointed out that in a population with $N_{c}$ clones there must be at least $N_{c}$ mutations (one in each clone) before $B$ can spread to fixation in the entire species. $N_{c}=2$ in our population. Let us assume $b \rightarrow B$ occurs in the $A$ clone. It will increase in frequency and will take with it allele $A$. That is $p$ will increase and $q$ decrease. As $q$ decreases the fitness of $a$ will increase and eventually $A B$ will cease to spread. Suppose at this point that the fitness of $a=1$ and that of $A=1-m$. The fitness of the $a b$ clone is now $1 \times(1-s)$ i.e., $1-s$ and this is equal to that of the $A B$ clone which is $(1-m) \times 1$ i.e., $1-m$. The latter clone has gained the advantageous mutation $B$ but its genetic load has not reduced. This follows because its fitness before the mutation occurred was $1-s$. After $B$ spread to fixation in the $A$ clone its fitness became $1-m$. As $1-s=1-m$ the fitness of the $A$ clone has not changed. It has gained in numbers but not in fitness. What of the $a$ clone? It has lost in numbers but not in fitness. Its fitness remains at $1-s$ as it was before the occurrence of $B$ in the $A$ clone. $B$ must go to fixation in both clones before the genetic load is removed. Therefore a favourable mutation must go to fixation in all clones of an asexual species before the genetic load created by the environmental change can be eliminated. If the species is intensely polymorphic, many of the clones will consist of a few individuals. Mutation rates are usually low. It follows that a very long period of time will elapse before a favourable mutation occurs in a small clone.

The problem of mutation loss may also be mentioned. Many favourable mutations are lost by chance before they reach fixation. Suppose a favourable mutation $b \rightarrow B$ arises. The fitness of $b$ is 1 and that of $B$ is $1+s$. Haldane (1927) has shown that the probability of $B$ reaching fixation is $2 s$ if $s$ is fairly small. Now Fisher (1930) has emphasised that mutants with large effects have little chance of being favourable. Therefore $s$ may often be of the order of say 0.01 . For every such mutant that is fixed 49 others are lost. Consider now an asexual species with $N_{c}$ clones. For each clone the number of mutants lost per mutant fixation is $(1 / 2 s)-1$. With $N_{c}$ clones there are $N_{c}(1 / 2 s)-N_{c}$ mutants lost before fixation occurs in the whole species. For a comparable sexual species $(1 / 2 s)-1$ mutations are lost per fixation. If $s=0.01$ about 49 mutations are lost in the sexual group, but with $N_{c}=10$ there are 490 lost from the asexual species.

Suppose now the environment changes continuously so that a series of mutations $b \rightarrow B, c \rightarrow C, d \rightarrow D, \ldots$ become favourable. What are the consequences for our multi-clonal asexual species? When $b \rightarrow B$ occurs the probability that the event is in an individual carrying $A$ is $p$. If it does so the spread of $B$ will increase $p$. The mutation $c \rightarrow C$ may then occur before $B$ has occurred in the depleted clone $a$. Again $C$ will tend to occur in the enlarged clone $A$, which will increase further in size at the expense of clone $a$. Continuous evolution may therefore reduce clone $a$ to very small numbers and it could eventually be lost from the population by drift. What 
will be left is a monomorphic population which is ill-adapted to the environment in the sense that it does not possess allele $a$. Mutation may reintroduce $a$ but it will tend to be eliminated again.

Loss of variation in the above way does not occur in sexual populations. Thus a mutation $b \rightarrow B$ which occurs in an individual carrying $A$ can spread through the entire population because recombination will create $a B$ gametes. The values of $p$ and $q$ are not affected and continuous evolution may occur without the loss of $a$.

\section{Deleterious mutations}

We are concerned here with the effect of Muller's ratchet (Muller, 1964) on a polymorphic asexual species. Under the influence of recurrent deleterious mutation and selection, a population at equilibrium consists of individuals with $0,1,2, \ldots$ mutations. This deterministic equilibrium is identical for asexual and sexual populations (Haigh, 1978; Maynard Smith, 1978). However, if the former loses its optimal class (i.e., the class with the lowest number of mutations) by drift, then it may not be regenerated. The ratchet has moved one notch. Recombination (i.e., sex) prevents this. The speed at which the ratchet moves is dependent on the number in the optimal class. The smaller the class the faster the ratchet. The size of the optimal class is

$$
n_{0}=N e^{-u / s}
$$

where $u$ is the mutation rate per genome and $s$ the selective disadvantage of each mutation so that an individual with $k$ mutations has a fitness $(1-s)^{k}$. Now in our polymorphic population $n_{0}$ for clone $A$ is

and for clone $a$ it is

$$
n_{0} A=(N p) e^{-u / s}
$$

$$
n_{0} a=(N q) e^{-u / s} .
$$

As $p>q$ it follows that $n_{0} A>n_{0} a$ (Manning $1982 b ; 1983$ ). This means the ratchet moves more quickly in clone $a$ than clone $A$. What are the consequences of this? Manning (1982 $b$ ) has argued that a rapid accumulation of deleterious mutations in clone $a$ will force down $q$ and increase $p$. The initially less common allele will be further depressed in frequency. If the environment changes so that $a$ increases in frequency and $p<q$ then clone $A$ will, in its turn, accumulate deleterious mutations. Frequent reversals in the relationship $p>q$ will result in both clones irreversibly aquiring deleterious mutations by this hitch-hiking mechanism (Manning, 1983).

The following argument assumes the relationship $p>q$ is not often reversed. That is, the environment is relatively constant (a characteristic which seems to favour sex, Ghiselin, 1974; Maynard Smith, 1978). Because $p>q$ it follows that $n_{0} A>n_{0} a$. Clone $a$ will accumulate deleterious mutations $n_{0} A / n_{0} a$ times more rapidly than clone $A$. That is, the mean fitness of clone $a$ will reduce below that of clone $A$. As a result assume that $p$ increases to $p 1$ and $q$ reduces to $q 1$. This will bring a consequent reduction in the fitness of clone $A$, as allele $A$ reduces in fitness and allele $a$ increases 
in fitness when they move away from their equilibrium values. However, $n_{0} A$ is now

$$
n_{0} A 1=(N p 1) e^{-u / s}
$$

and $n_{0} a$ is now

$$
n_{0} a 1=(N q 1) e^{-u / s} \text {. }
$$

As $p 1>p$ and $q 1<q$ it follows that $n_{0} A / n_{0} a<n_{0} A 1 / n_{0} a 1$. That is the ratchet will move less rapidly in clone $A$ and even more rapidly in clone $a$. The increase in $p$ and reduction in $q$ will continue, bringing with it a decrease in the fitness of clone $A$ as a result of frequency dependent selection. Eventually clone $a$ will reduce to a few individuals. At this point it may still have the same fitness as clone $A$ as the latter is far above its equilibrium value. Clone $a$ may then be lost by drift leaving a population which is poorly adapted in the sense that it lacks allele $a$. Therefore Muller's ratchet has a powerful short-term effect which is similar to, and is reinforced by, the long-term effect of continuous evolution.

\section{Discussion}

It is now well known that sexual populations are usually intensely variable. Electrophoresis has revealed high percentages of polymorphic loci (Lewontin, 1974). This technique does not detect all variation so that it is probable that most loci in most populations are polymorphic. A comparable asexual population would be divided into a very large number of clones, each of small size. In each clone favourable mutations would be acquired painfully slowly and the ratchet would move rapidly. In many of the clones numbering tens or hundreds of individuals $n_{0}<1$ will be commonplace. Haigh (1978) argued that in such a clone the rate of accumulation of deleterious mutations will be greater than it can bear. It will be lost. For clones to be maintained in asexual populations they need to be large. This model indicates an answer to the observation that asexual species are often very numerous with a wider geographical distribution than their bisexual competitors (White, 1973). A large population may be a prerequisite for their existence.

Finally how should we compare the fitness of competing sexual and asexual species? The most meaningful comparison is between that of clone and a particular allelic combination. The latter has the low mutation load and lag load of its entire species. The former has levels of load controlled directly by its size. In small clones this should be devastatingly high. So high their long-term and short-term future would be very insecure.

\section{REFERENCES}

FISHER, R. A. 1930. The Genetical Theory of Natural Selection. Oxford University Press, Oxford.

GHISELIN, M. T. 1974. The Economy of Nature and the Evolution of Sex. University of California Press.

HALDANE, J. B. S. 1927. A mathematical theory of natural and artificial selection. Part V. Selection and Mutation. Proc. Camb. Phil. Socy., 23, 838-844.

MANNING, J. T. 1981. The "survivor effect" and the evolution of parthenogenesis and self-fertilization. J. Theor. Biol., 93, 491-493. 
MANNING, J. T. 1982a. Sex and the fixation of single favourable mutations. J. Theor. Biol, 94, 905-908.

MANNING. J. T. 1982b. Recombination maintains equilibrium frequencies of common alleles. Heredity, 49, 253-254.

MANNING, J. T. 1983. Hitch-hiking and a short-term advantage for sex. J. Theor. Biol,, in press. MA YNARD SMITH, J. 1976. What determines the rate of evolution? Am. Natur., 110, 331-338. MAYNARD SMITH, J. 1978. The Evolution of Sex. Cambridge University Press, Cambridge. WhITE, M. J. D. 1973. Animal Cytology and Evolution. Cambridge University Press, Cambridge. 